\title{
Editorial
}

Theme: Printing and Additive Manufacturing

Guest Editors: Niklas Sandler and Jukka Rantanen

\section{Printing and Additive Manufacturing}

\author{
Jukka Rantanen ${ }^{1,3}$ and Niklas Sandler ${ }^{2,3}$
}

Received 20 May 2019; accepted 20 May 2019; published online 23 July 2019

\section{EDITORIAL AAPSPT}

by Jukka Rantanen and Niklas Sandler

Additive manufacturing (AM) of pharmaceutical products is a potential game-changer for the whole pharmaceutical business area. This opens totally new possibilities for implementing innovative product design principles. For instance, the possibility to design the inner structure of a product gives unique opportunities to tailor-make the products size and shape, as well as inner structure of it and, by this, means to control the drug release characteristics. This approach is paving the way towards patient-centered medication strategies and implementation of mass customization principles.

Many of the potential AM methods are naturally operating the continuous mode, which is underpinning the need for early implementation of quality by design (QbD) and process analytical technologies (PAT) principles. Additionally, AM methods are typically based on computer-based design of the product geometry giving a unique possibility to early implementation of computational methods covering product design in a broader sense. Another important aspect is the materials science dimension; more fundamental work is needed to develop materials and analytical methods that can be utilized when implementing AM principles.

As a summary, the pharmaceutical area is still missing broadly accepted processing principles, key equipment, optimized excipients, computational methods, and quality control tools for additive manufacturing of innovative pharmaceuticals. This theme issue is collecting recent scientific development related to these aspects and, by this, means

\footnotetext{
Guest Editors: Niklas Sandler and Jukka Rantanen

${ }^{1}$ Department of Pharmacy, University of Copenhagen, Copenhagen, Denmark.

2 Åbo Akademi University, Turku, Finland.

${ }^{3}$ To whom correspondence should be addressed. (e-mail: jukka.rantanen@sund.ku.dkniklas.sandler@abo.fi)
}

creating a scientific basis for innovative pharmaceutical products for the twenty-first century.

In this theme issue, we have an excellent collection of cutting edge contributions from the field exemplifying the use of printing technologies in manufacturing of drug delivery systems. The journal articles include intriguing examples of the use of advanced materials in three-dimensional structures for tailoring release and dose adjustment for biologicals and small molecules.

We are delighted to see progress in the application of printing technologies. However, further development will be needed for the 3D technology to establish itself as a multipurpose tool for future treatments and delivery systems. Printing technologies will be able to become fabrication tools of the future if state-of-the-art printers are continuously developed, processing speeds are improved, and wider range of printable materials are developed to broaden the possibilities to create multifunctional drug delivery systems and medical devices of the future.

It is probably fair to say that the development of printing processes in the pharmaceutical field has moved from its infancy to adolescence, and the next decade will most likely show immense progress and activity within the field including the pharma industry, various startups, pharmacies, and hospitals, where point-of-care fabrication in hospitals can be reality, and individualized treatments are made closer to the patient. Safe integration of these products into the healthrelated big data and internet of things (IoT) remains one of the future challenges.

Publisher's Note Springer Nature remains neutral with regard to jurisdictional claims in published maps and institutional affiliations. 